\title{
Conversing with a Devil's Advocate: Dialogical Dynamics of Movement and Speech in Deception and Conflict
}

Nicholas Duran ${ }^{\star}$ and Riccardo Fusaroli**

*Arizona State University; ${ }^{* *}$ Aarhus University

\section{Rationale}

- A novel multi-modal analysis to reveal unique patterns of interpersonal coordination as a function of the sociocognitive demands of deception; in the context of agreement and disagreement conversations.

- Synergistic interdependence: examines aspects of continuous movement and speech; co-regulatory patterns of speech, shared between interacting partners.

- Contrasted two hypotheses, "coordination as":

- "spontaneous affiliation," suggests lower degree of coordination in deceptive and non-affiliative (disagreement) conversations.

-modulated by intentional goals," suggests deceptive conversations involve attentive monitoring engagement of the deceived; give rise to higher coordinated behavior at the dyadic level; unrelated to rapport.

\section{Study}

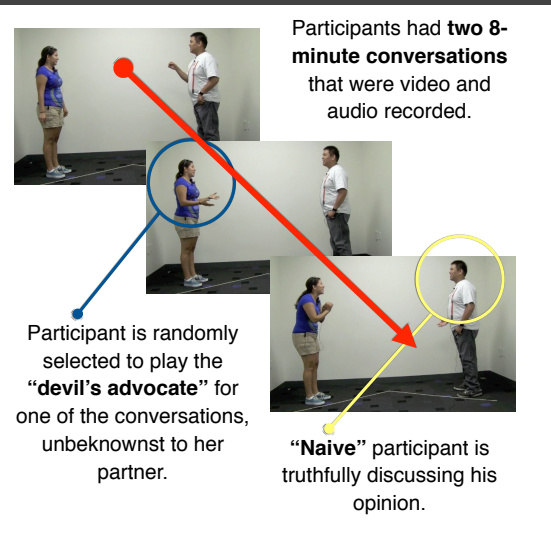

Method: Prior to interaction, participants' true opinion on 10 controversial topics were collected; two topics selected, one in which they disagreed and another in which they agreed; depending on condition, for one conversation, the devil's advocate took an opposite opinion of what he/she actually believes (deception).

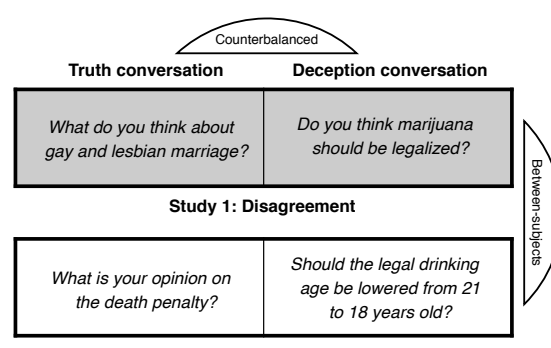

Study 2: Agreement

After each conversation, ratings provided by each participant concerning interactional rapport.

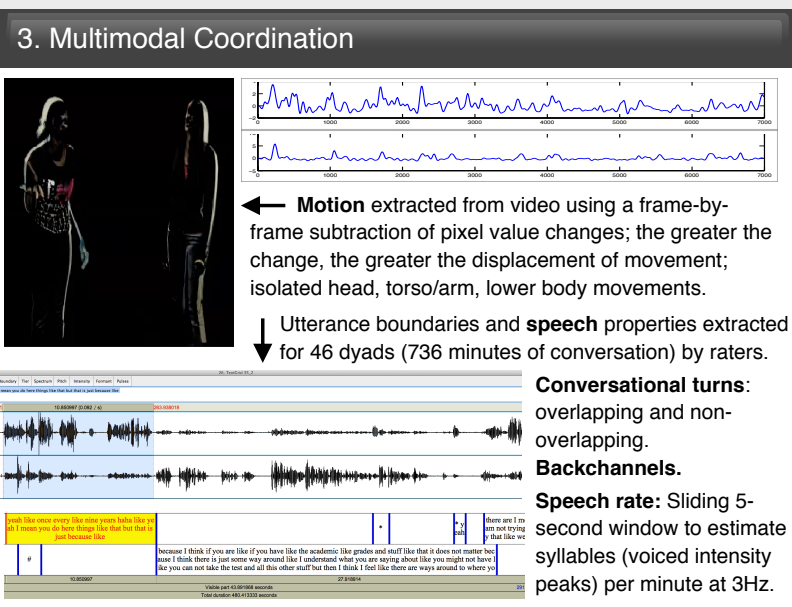

4. Predictions and Analysis

\begin{tabular}{|c|c|c|c|c|}
\hline & \multirow{2}{*}{\multicolumn{2}{|c|}{$\begin{array}{c}\text { affiliation } \\
\begin{array}{c}\text { Lower } \\
\text { coordination }\end{array}\end{array}$}} & $\begin{array}{c}\text { Intentional } \\
\text { goals }\end{array}$ & \multirow{3}{*}{$\begin{array}{l}\text { Prediction: Low-level } \\
\text { coordination as a } \\
\text { function of speaker's } \\
\text { intentions, not merely } \\
\text { mechanism of affiliation } \\
\text { or cooperation. }\end{array}$} \\
\hline Deception vs. truth & & & $\begin{array}{c}\text { Higher } \\
\text { coordination }\end{array}$ & \\
\hline $\begin{array}{c}\text { Disagreement vs. } \\
\text { agreement }\end{array}$ & $\begin{array}{c}\text { Lower } \\
\text { coordinatio }\end{array}$ & & No prediction & \\
\hline 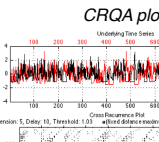 & Wordent: & $\begin{array}{l}\text { Anal } \\
\text { analy } \\
\text { corre } \\
\text { analy } \\
\text { quan }\end{array}$ & $\begin{array}{l}\text { lysis: The } \mathbf{n} \\
\text { yzed with wi } \\
\text { elation (WLC } \\
\text { yzed with cr } \\
\text { tification an }\end{array}$ & $\begin{array}{l}\text { lotion time series } \\
\text { ndowed-lagged cross } \\
\text { C); speech time series } \\
\text { oss-recurrence } \\
\text { alysis (CRQA): }\end{array}$ \\
\hline & & $D V$ & Coordination & Index characterization \\
\hline & & RR & Amount & $\%$ of repeating values \\
\hline & & DET & Stability & \% extended repeating values \\
\hline & & L & Stability & avg. length of repeat \\
\hline & & LMAX & Stability & length longest repeat \\
\hline & & ENTR & Complexity & variance of repeat \\
\hline & & T1 & Stability & avg. distance between repeat \\
\hline
\end{tabular}

\section{Results}

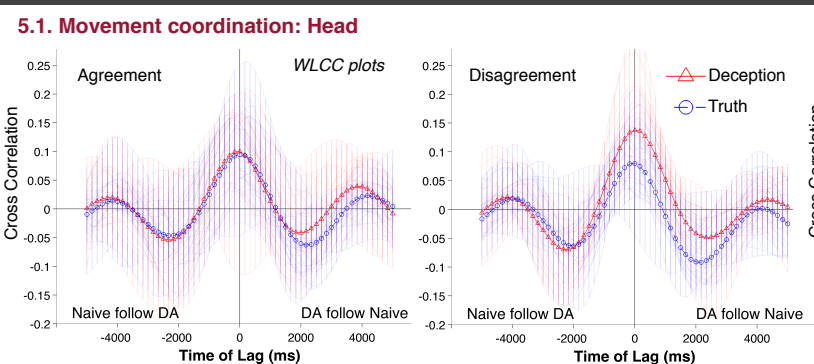

4 In WLCC plots, greatest coordination revealed at near-synchronous

moments, decreasing as alignment was lagged; in interaction plot, for truth conversations, peak coordination greatest for agreement; for deception, peak coordination much greater for disagreement; the pattern holds across all lags up to $1000 \mathrm{~ms}$; in surrogate pair comparison, no statistically significant pettems between conditions.

5.2. Speech coordination: Speech Rate
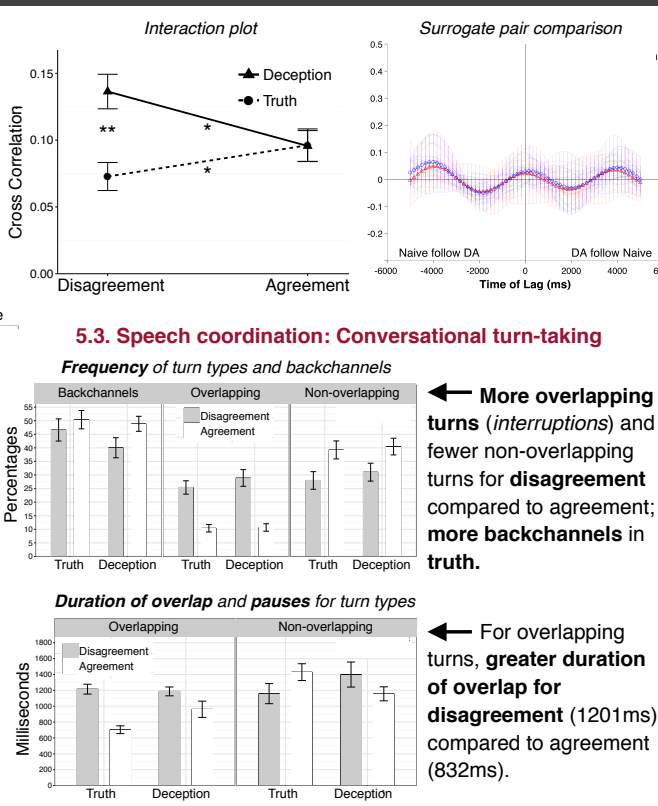

5.4. Ratings of interactional rapport

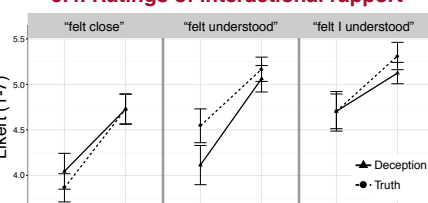

5. Machine learning and generalizabilty

5 -fold feature selection and crossvalidation process to predict deception and conflict; deception: less head synchrony at lag -3 seconds (DA follows), 61.17\%; disagreement. lower speech rate (RR \& LMAX), higher overlapping Disagree Agree Disagree Agree Disagree
Agreement showed higher ratings of rapport; deception (compared to truth) showed $74.97 \%$
Surrogate pair comparison

5.3. Speech coordination: Conversational turn-taking Frequency of turn types and backchannels

Duration of overlap and pauses for turn types

AS

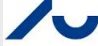

AARHUS UNIVERSITY

- Major conclusions: Support found for primary prediction; but also requires more nuanced view of multifunctional coordination. For spontaneous affiliation and conflict: speech rate measures and turn-taking showed less coordination for disagreement; interaction with movement and conflict revealed less movement with truth and disagreement; also significantly less rapport. For
modulated by intentional goals: more structured speech rate coordination for deception; critical interaction with head movemen and conflict revealing greater coordination in deception and disagreement; also significantly less rapport.

- Future directions: Linguistic and content analysis; comparison of individual- and dyadic-level behaviors. 